\title{
Stress-constrained topology optimization for compliant mechanism design
}

de Leon, Daniel M.; Alexandersen, Joe; Jun, Jun S.; Sigmund, Ole

Published in:

Structural and Multidisciplinary Optimization

Link to article, DOI:

$10.1007 / \mathrm{s} 00158-015-1279-\mathrm{z}$

Publication date:

2015

Document Version

Peer reviewed version

Link back to DTU Orbit

Citation (APA):

de Leon, D. M., Alexandersen, J., Jun, J. S., \& Sigmund, O. (2015). Stress-constrained topology optimization for compliant mechanism design. Structural and Multidisciplinary Optimization, 52(5), 929-943.

https://doi.org/10.1007/s00158-015-1279-z

\section{General rights}

Copyright and moral rights for the publications made accessible in the public portal are retained by the authors and/or other copyright owners and it is a condition of accessing publications that users recognise and abide by the legal requirements associated with these rights.

- Users may download and print one copy of any publication from the public portal for the purpose of private study or research.

- You may not further distribute the material or use it for any profit-making activity or commercial gain

- You may freely distribute the URL identifying the publication in the public portal

If you believe that this document breaches copyright please contact us providing details, and we will remove access to the work immediately and investigate your claim. 


\title{
Stress-constrained topology optimization for compliant mechanism design
}

\author{
Daniel M. De Leon • Joe Alexandersen • \\ Jun S. O. Fonseca • Ole Sigmund
}

Received: date / Accepted: date

\begin{abstract}
This article presents an application of stressconstrained topology optimization to compliant mechanism design. An output displacement maximization formulation is used, together with the SIMP approach and a projection method to ensure convergence to nearly discrete designs. The maximum stress is approximated using a normalized version of the commonly-used pnorm of the effective von Mises stresses. The usual problems associated with topology optimization for compliant mechanism design: one-node and/or intermediate density hinges are alleviated by the stress constraint. However, it is also shown that the stress constraint alone does not ensure mesh-independency.
\end{abstract}

Keywords topology optimization · compliant mechanisms $\cdot$ stress constraints

This work was performed during the first author's visit to the Department of Mechanical Engineering at the Technical University of Denmark supported by the CNPq scholarship, process number 211610/2013-5. The second and fourth authors were supported by the Villum Foundation through the NextTop project.

D. M. De Leon · J. S. O. Fonseca

Department of Mechanical Engineering, Applied Mechanics Group (GMAp), UFRGS - Federal University of Rio Grande do Sul, Sarmento Leite, 425, 90050-170, Porto Alegre, RS, Brazil

E-mail: danielmdeleon@gmail.com

E-mail: jun@ufrgs.br

J. Alexandersen · O. Sigmund

Department of Mechanical Engineering, Solid Mechanics,

Technical University of Denmark, Niels Koppels Allé,

Building 404, 2800, Kongens Lyngby, Denmark

E-mail: joealex@mek.dtu.dk

E-mail: sigmund@mek.dtu.dk

\section{Introduction}

For compliant mechanisms, elastic strain is the basis for kinematic actuation. Their main advantages are reduced wear, friction and backlash compared to conventional mechanisms. These characteristics lead to higher precision and reliability to perform the tasks they are built for. Moreover, they can use their own stored elastic energy to return to the initial position when the input actuation is ceased, without the need for a second actuation (Howell, 2013). The main disadvantages are: part of the input work being spent on elastic deformation of the mechanism itself, susceptibility to fatigue and the lack of systematic design methodologies for real-life applications.

Compliant mechanisms are widely used as precision mechanical devices in the biomedical area and in MEMS - MicroElectroMechanical Systems. MEMS are built in very small sizes, using advanced micro-scale manufacturing; they are usually etched in a single material layer with subsequent underetching.

A mechanism design procedure starts by the specification of the available design domain, the supports, the input and output ports and their forces and kinematics, the strength requirements, and the manufacturing constraints, which may include length scales and manufacturability issues. A design problem formulation should include all this information, and also be reasonably independent of numerical parameters such as numerical discretization and solution algorithms.

Among several techniques applied in compliant mechanisms design, topology optimization (Sigmund, 1997; Frecker et al., 1997) has been one of the most general and systematic, since it has been able to deal with the precise elastic behavior of complex geometries. As the goal of this work is not to provide a historical back- 
ground on the subject, only the most relevant formulations are discussed in the following.

Some of the first works applying topology optimization to compliant mechanisms design by Sigmund (1997) and Larsen et al. (1997), used a density-based formulation and defined the objective function as maximization of the output port displacement. This formulation yields energy efficient designs, however with lumped compliant regions or one-node-connected hinges connecting stiff members, thus effectively mimicking rigidbody mechanisms. This formulation has served as a basis to many others since its publication, including the present work. Whereas the original works considered linear (small) displacements, the concept has also been extended to finite deformations (Pedersen et al., 2001). Although the inclusion of finite deformation theory is imperative for practical applications, the focus in the present work is on the inclusion of stress constraints rather than on practical applications and hence uses simpler linear elasticity theory. However, it is expected that the conclusions drawn here for the linear problem largely apply to the non-linear case as well.

In the topology optimization method, a well-known challenge is to avoid numerical instabilities (Sigmund and Petersson, 1998) and ensure convergence with meshrefinement. In order to obtain mesh-independent and checkerboard-free designs, filtering techniques are by far the most common procedures. Both sensitivity filtering (Sigmund, 1997) and density filtering (Bourdin, 2001; Bruns and Tortorelli, 2001), successfully produce designs for a chosen length scale (filter radius), although with the appearance of an intermediate density (gray) transition area between the solid and void regions of the domain and the appearance of one-node-connected and/or gray-scale localized hinge regions. In order to overcome the former difficulties, various projection schemes have been proposed (see Guest et al., 2004; Sigmund, 2007; Guest, 2009, among others). Depending on threshold values, these filters may control minimum feature sizes of either solid or void regions, however, they do not prevent localized artifacts (Wang et al., 2011) which for compliant mechanism design show up in the form of one-node-connected hinges.

The question of whether lumped or distributed compliant mechanisms are optimal is a long standing and still open question in the field. One issue is certain, however. The appearance of one-node-connected hinges in topology optimization of compliant mechanism must be avoided since it represents poor finite element modeling. To ensure satisfactory stress solution quality, any feature in a geometry should be discretized by at least a couple of elements.
Several schemes have been proposed to alleviate the one-node-connected hinge problem. The schemes can roughly be divided into (local) geometry-based and (global) energy-based formulations. It is not the goal of the present paper to provide a complete review of all these papers and techniques. Instead we just mention a few of the most recently published approaches:

- a robust formulation for compliant mechanisms proposed by Sigmund (2009) and Wang et al. (2011), that succeeds in imposing length scale, alleviating the hinge problem and taking under- and/or overetching in the manufacturing process into account. Despite the higher computational time compared to other formulations (two to three finite element analyses have to be performed at each iteration), it succeeds in obtaining compliant mechanisms, devoid of one-node-connected hinges and with an allowed error bound for manufacture.

- a strain-based multi objective formulation proposed by Lee and Gea (2014), where the effective strain is included in the objective function;

- a compliance-based multi-objective function proposed by Zhu et al. (2014), using a weighted function consisting of the input and output port compliances;

The field of compliant mechanism design is not restricted to the density approach used in this paper, but has also been studied by means of level-set methods, (e.g. Luo et al., 2008), and phase-field methods (Takezawa et al., 2010). Among them, one can cite level-set based formulations with a geometric penalty on the curvature to avoid small features (Luo et al., 2008), a formulation for minimum stress design (Allaire and Jouve, 2008) and a formulation that minimizes a functional using a relation between the minimum compliance of the output and input ports and with a chosen target for the geometrical advantage of the mechanism (Zhu and Zhang, 2012).

Although the above procedures somewhat successfully avoid one-node-connected hinges, we here postulate that the optimal mechanism layout is neither ensured by purely energy-based, nor by purely geometrybased measures. The avoidance of localized hinges should come from a mechanics-based reformulation of the compliant mechanism optimization formulation in terms of local field measures, possibly combined with some measures to avoid mesh-dependency. One way of doing this is to include a maximum stress limit as suggested in this paper.

It is noticeable from the available literature that a steady progress has been made in addressing the convergence of the numerical implementation of kinematicsonly formulations, but the strength requirement has been largely absent. A natural explanation for this is 
that the stress constraint problem is still an open research area - even for simpler non-mechanism design problems (e.g. Allaire et al., 2004; Le et al., 2010), among others. To the best of our knowledge, stressconstrained mechanism design formulations have only been formulated for truss-based mechanism design by Saxena and Ananthasuresh (2001) and in a preliminary work by Meneghelli and Cardoso (2013).
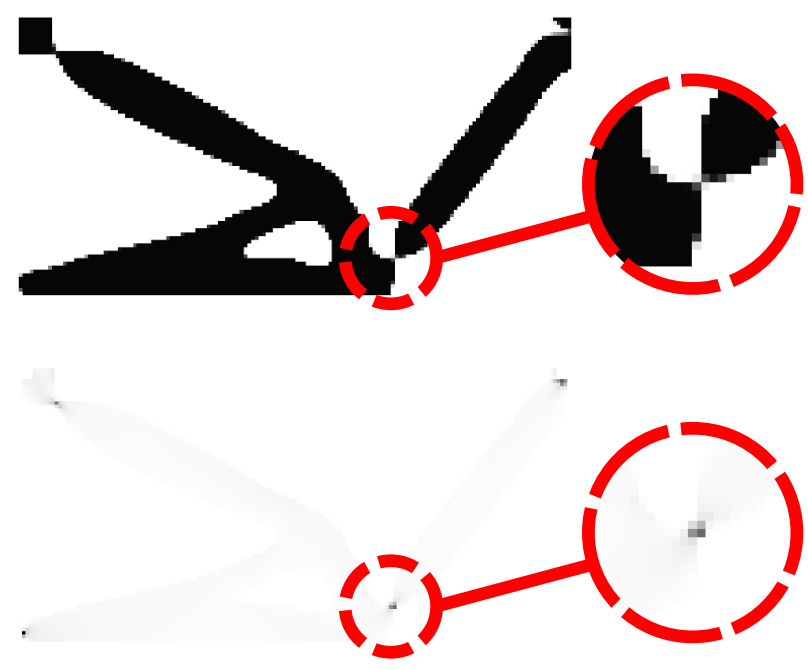

Fig. 1 A typical optimized compliant inverter design with hinged connections and the corresponding grey scale stress distribution below with black denoting high and white denoting low stresses. The circles magnify a hinge and its corresponding stress concentrations.

This paper applies stress-constrained topology optimization to compliant mechanism design. The additional constraint is applied to a normalized global stress measure, based on the well-known p-norm of the von Mises effective stress (Duysinx and Sigmund, 1998; Le et al., 2010). The goal of the stress constraint is to ensure mechanically sensible mechanisms by alleviating the one-node-connected hinge problem and lowering the general stress level, in turn improving the fatigue life of the device. Fig. 1 shows the von Mises stress plot of a typically one-node-connected hinge compliant mechanism with the distinctive stress concentration at the hinge.

The paper is organized as follows. Section 2 introduces the general topology optimization formulation for compliant mechanism design including projection filtering, the stress-norm constraint and a discussion of appropriate continuation strategies. Section 3 demonstrates the procedure applied to the compliant inverter and the compliant gripper benchmark problems. The section includes various parameter studies such as convergence with mesh-refinement, dependency on stress constraint value and output stiffness. Section 4 con- cludes the work and discusses options for further studies.

\section{Optimization problem}

A mechanism is expected to react when a force is acting upon it. Besides that, it is required to perform work on a workpiece. The points where the external force is applied and where force is expected to react, we refer to as the input and output ports, respectively. Fig. 2 shows the general problem for topology optimized mechanism design. The physical domain, $\Omega=\Omega_{d} \cup \Omega_{s} \cup \Omega_{v}$, is split into three parts: the design domain, $\Omega_{d}$; a prescribed solid domain, $\Omega_{s}$; and a prescribed void domain, $\Omega_{v}$. The domain boundary, $\Gamma=\Gamma_{D} \cup \Gamma_{N}$, is made up of two parts: the Dirichlet boundary with prescribed displacements, $\Gamma_{D}$; and the Neumann boundary with zero normal stress, $\Gamma_{N}$. The input port is subjected to a given load, $f_{i n}$, and the output port exhibits the desired output, $u_{\text {out }}$, due to elastic deformation of the domain.

Most actuators have a built-in stiffness that needs to be taken into account, together with the workpiece stiffness. To physically describe these, springs $k_{i n}$ and $k_{\text {out }}$, are added to the input and output ports, respectively, as shown in Fig. 2. Alternatively, input and output displacements can be used as delimiters, imposing a constraint on the displacements.

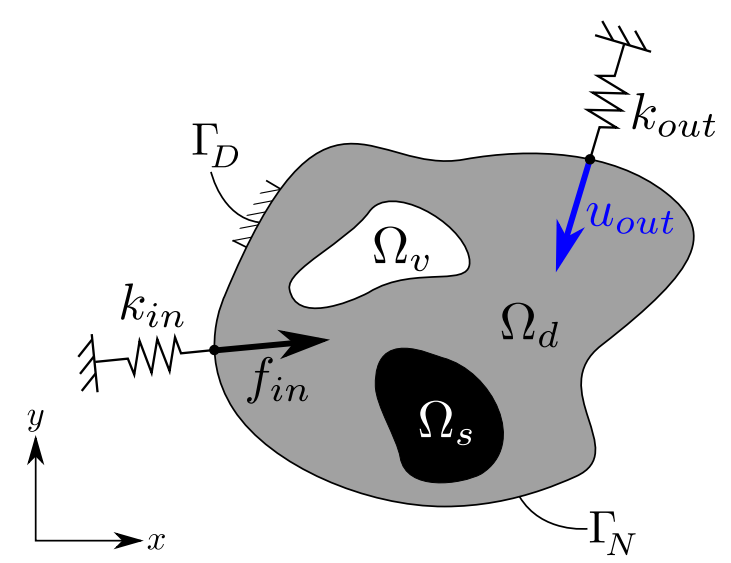

Fig. 2 General problem for mechanism design.

The presented work builds on the formulation by Sigmund (1997), where the objective function is written as minimization of displacement in the output port subject to the equilibrium equations and a volume constraint. The main contribution of this work is the addition of a constraint on the maximum stress to the 
optimization problem. The general formulation is given as:

$$
\begin{aligned}
\min _{\boldsymbol{\rho}} & : f(\boldsymbol{\rho})=\mathbf{l}^{T} \mathbf{u} \\
\text { s.t. } & : \mathbf{K}(\boldsymbol{\rho}) \mathbf{u}=\mathbf{f} \\
& : f_{v}(\boldsymbol{\rho})=\frac{\sum_{i \in \mathbb{N}_{e}} \overline{\tilde{\rho}}_{i}(\boldsymbol{\rho}) v_{i}}{V} \leqslant V^{*} \\
& : f_{s}(\boldsymbol{\rho})=\max (\boldsymbol{\sigma}) \leqslant \sigma^{*} \\
& : 0 \leqslant \rho_{i} \leqslant 1, \quad i \in \mathbb{N}_{e}
\end{aligned}
$$

where $\mathbf{K}$ is the stiffness matrix obtained by means of finite element discretization, $\mathbf{u}$ and $\mathbf{f}$ are the solution and the input vectors respectively, $\boldsymbol{\rho}$ is the vector of design variables, $\mathbb{N}_{e}$ is the set containing all elements and $\overline{\tilde{\rho}}_{i}$ is the physical density associated with the $i$ 'th design element. The vector $\mathbf{l}$ takes zero in all positions except for the position corresponding to the output degree of freedom, which is set to one. The total volume of the design domain is $V, V^{*}$ is the allowed volume fraction and $v_{i}$ is the volume of the $i$ 'th element. The vector $\boldsymbol{\sigma}$ contains the element stress measures, to be defined in section 2.2, and $\sigma^{*}$ is a chosen threshold for the maximum elemental stress.

The element stiffness matrices $\mathbf{K}_{i}$ are calculated as $\mathbf{K}_{i}=E_{i} \mathbf{K}_{\mathbf{0}}$ where $\mathbf{K}_{\mathbf{0}}$ is the element stiffness matrix for unit material stiffness and $E_{i}$ is the material stiffness obtained using the well-known solid isotropic material interpolation with penalization (SIMP):

$E_{i}=E_{\min }+\overline{\tilde{\rho}}_{i}{ }^{k}\left(E_{0}-E_{\min }\right)$

where $E_{0}$ is the stiffness of the solid phase, $E_{\min }$ is a small stiffness attributed to regions to avoid ill-conditioning and $k$ is the penalization parameter. Adjoint sensitivity analysis can be applied to calculate the derivative of objective function with respect to the physical densities:

$\frac{\partial f}{\partial \tilde{\tilde{\rho}}_{i}}=\lambda^{T} \frac{d \mathbf{K}}{d \tilde{\tilde{\rho}}_{i}} \mathbf{u}$

where $\boldsymbol{\lambda}$ is the adjoint vector calculated from $\mathbf{K} \boldsymbol{\lambda}=-\mathbf{l}$.

In this work, an implementation of MMA (Method of Moving Asymptotes) (Svanberg, 1987) written in C is used as the mathematical programming algorithm.

\subsection{Projection filtering}

Considering the optimization problem as stated in (1) with the physical densities, $\overline{\tilde{\boldsymbol{\rho}}}$, set equal to the design variables, $\boldsymbol{\rho}$, will lead to two well-known problems; the formation of checkerboard patterns and mesh-dependent designs (Sigmund and Petersson, 1998). The first is due to bad numerical modeling from low-order discretization of the design (Díaz and Sigmund, 1995). The second problem means that instead of getting higher resolution images of the topology, the optimization process starts to add small details in the solid and/or void regions. This leads the optimization procedures to different topologies, creating a dependence on mesh size.

Selecting the filtered densities, $\tilde{\boldsymbol{\rho}}$, to be the new physical densities leads to another problem; the appearance of gray transition areas between solid and void phases. These intermediate densities can be disregarded by simple thresholding for simpler problems such as compliance minimization. Unfortunately, however, in more complex problems such as compliant mechanisms design, this postprocessing may lead to wrong physical interpretations.

A recent tool that has been widely used to avoid gray transition areas is the implementation of projection methods (Guest et al., 2004; Sigmund, 2007). All the filtered density values above a threshold $\eta$ are projected to 1 and values below it to 0 . As discussed in the introduction, simple projection filtering does not avoid one-node-connected hinges - this would require a robust formulation (Sigmund, 2009; Wang et al., 2011). Here, however, we refrain from using the robust formulation since we want to investigate whether the added stress constraint will avoid the one-connected-hinge issue as well as provide mesh-independency without further means.

To ensure differentiability, the projection method applies a smoothed Heaviside funtion:

$\overline{\tilde{\rho}}_{i}=\frac{\tanh (\beta \eta)+\tanh \left(\beta\left(\tilde{\rho}_{i}-\eta\right)\right)}{\tanh (\beta \eta)+\tanh (\beta(1-\eta))}$

where $\beta$ determines the slope (sharpness) of the curve and $\eta$ the threshold value.

The projected variables, $\overline{\tilde{\boldsymbol{\rho}}}$, are chosen as the physical density variables. They are a function of the filtered densities, $\tilde{\boldsymbol{\rho}}$, and the design variables, $\boldsymbol{\rho}$, and thus the sensitivities are corrected using the chain rule as detailed by Sigmund (2007). The discreteness of optimized topologies is measured by a gray-level indicator as introduced by Sigmund (2007):

$M_{n d}=\frac{\sum_{i=1}^{N} 4 \overline{\tilde{\rho}}_{i}\left(1-\overline{\tilde{\rho}}_{i}\right)}{N} \times 100 \%$

When $\overline{\tilde{\rho}}_{i}=0.5$ for all elements, the value of $M_{n d}$ is $100 \%$, and when $\overline{\tilde{\rho}}_{i}=0$ or $\overline{\tilde{\rho}}_{i}=1$ for all elements, the value of $M_{n d}$ is $0 \%$.

\subsection{Stress constraints}

Stress-based topology optimization is a standing challenge. Applying stress constraints to a given problem 
raises additional obstacles to be overcome. Firstly, the singularity problem appears when elements that approach zero density vanish from the design space, resulting in degenerated subspaces impossible to be identified by the algorithm, that converges to local minima. The problem was first encountered and clearer to be noticed for optimization of truss structures (Kirsch, 1990; Cheng and Jiang, 1992), where the cross sectional area of some members tending to zero, degenerate the solution space. The problem with trusses is overcome by means of functions that smooth the feasible design space (Cheng and Guo, 1997; Rozvany and Sobieszczanski-Sobieski, 1992) and this generally allows the algorithm to converge to better local minima. Later, these functions were adapted to continuum problems (Duysinx and Bendsøe, 1998; Duysinx and Sigmund, 1998). In the present work, a relaxation scheme introduced by Le et al. (2010) is applied to the von Mises stress on each design element. The elemental stress measure for a given element becomes:

$\sigma_{i}=\overline{\tilde{\rho}}_{i}^{q} \sigma_{v m, i}$

where $\sigma_{i}$ is the relaxed stress measure, $\overline{\tilde{\rho}}_{i}$ is the element physical density, $q$ is a relaxation parameter and $\sigma_{v m, i}$ is the von Mises effective stress of the (solid) element centroid:

$\sigma_{v m, i}=\left(\boldsymbol{\sigma}_{i}^{T} \mathbf{V} \boldsymbol{\sigma}_{i}\right)^{\frac{1}{2}}$

with $\mathbf{V}$ being the auxiliary matrix:

$\mathbf{V}=\left[\begin{array}{ccc}1 & -\frac{1}{2} & 0 \\ -\frac{1}{2} & 1 & 0 \\ 0 & 0 & 3\end{array}\right]$

The element stress vector, $\boldsymbol{\sigma}_{i}=\left\{\sigma_{11}, \sigma_{22}, \sigma_{12}\right\}^{T}$, is calculated for fully solid material:

$\boldsymbol{\sigma}_{i}=\mathbf{C}_{0} \mathbf{B u}_{i}$

where $\mathbf{C}_{0}$ is the elasticity tensor for the base material, $\mathbf{B}$ is the strain-displacement matrix and $\mathbf{u}_{i}$ is a vector containing the $i$ 'th element's degrees of freedom of the global displacement vector.

The relaxation, equation (6), allows the elemental stress measure to go to zero when $\overline{\tilde{\rho}}_{i} \rightarrow 0$ and recovers the original stress when $\overline{\tilde{\rho}}_{i} \rightarrow 1$.

According to (Duysinx and Bendsøe, 1998) this model simplifies the computational effort for solving stress constrained problems. However, they also notice that the optimization problem solved is inconsistent with physics and can lead to artificial removal of material. This issue is fully addressed in the original paper. Nevertheless, since the projection filtering technique applied in this work removes intermediate densities successfully, this physical inconsistency is alleviated in the optimized results. A comparison between the optimized and interpreted results confirms this conclusion. The same formulation has with success been used in the work of Le et al. (2010) for compliance design.

The highly non-linear relation between stress levels and element density is another important issue to consider. Stress levels can vary significantly, especially when holes and corners are formed in the topology. The algorithm must be robust enough to handle these variations, otherwise it becomes difficult for the optimization to converge.

The last, but no less important, issue is the local nature of stress. As it is computationally intractable to impose one constraint on each finite element in the mesh, the most common way to deal with stress constraints is to select one global stress measure. In this work, the global p-norm stress measure proposed by Duysinx and Sigmund (1998) and later adopted and extended by Le et al. (2010) is applied in order to approximate the maximum stress of the design domain. This formulation aims to control the stress level by capturing the trend of the maximum stress. The stress constraint can be written as:

$\sigma_{P N}=\left(\sum_{i \in \mathbb{N}_{\sigma}} v_{i} \sigma_{i}^{p}\right)^{\frac{1}{p}}$

where $\mathbb{N}_{\sigma}$ is the set of elements to be constrained, $v_{i}$ is the elemental volume, $\sigma_{i}$ is the element relaxed stress measure and $p$ is the stress norm parameter. It can be proven that for $p \rightarrow \infty$, the p-norm approaches the maximum stress. However, the higher the value of $p$, the greater the degree of non-linearity and discontinuity and thus the greater the chances to find poor local minima. In this work, a value of $p=12$ has worked successfully.

The normalized stress measure as introduced by Le et al. (2010) is used in this work:

$\tilde{\sigma}_{P N}=c \sigma_{P N}$

The normalization parameter $c$ is proposed to give physical meaning to the p-norm and is defined for $n \geqslant$ 1 , where $n$ is the design iteration number, as:

$c^{n}=\alpha^{n} \frac{\sigma_{\max }^{n-1}}{\sigma_{P N}^{n-1}}+\left(1-\alpha^{n}\right) c^{n-1}$

where $\sigma_{\max }$ is the actual maximum element stress measure and $\alpha$ is a parameter that controls the update of $c$ between iterations. In this work, $\alpha^{n}=0.5$ for all $n$ and $c^{0}=1$ was used. The normalization provides a closer approximation of the maximum stress:

$\max (\boldsymbol{\sigma}) \approx \tilde{\sigma}_{P N}$ 
allowing for easier control of the maximum stress using a physical limit stress, $\sigma^{*}$. The normalized stress measure, equation (11), is chosen as the constraint function and the resulting optimization problem is thus as detailed in equation (1) but with $f_{s}=\tilde{\sigma}_{P N}$.

Basically, this formulation uses the well-known formulation stated by Sigmund (1997) and adds a constraint on the normalized global stress measure proposed by Le et al. (2010). The goal in imposing a constraint of the maximum stress, by means of a p-norm, is to avoid peaks in the mechanism stress field, indirectly avoiding the appearance of localized hinges in the design.

The adjoint sensitivity method is also applied to calculate the gradient of the stress constraint:

$$
\begin{aligned}
\frac{\partial f_{s}}{\partial \tilde{\tilde{\rho}}_{i}}= & c \sigma_{\text {sum }}{ }^{\frac{1}{p}-1} v_{i} \sigma_{i}^{p-1} q \overline{\tilde{\rho}}_{i}^{q-1} \sigma_{v m, i} \\
& +k \overline{\tilde{\rho}}_{i}^{k-1}\left(E_{0}-E_{m i n}\right) \boldsymbol{\lambda}_{i}^{\sigma T} \mathbf{K}_{0} \mathbf{u}_{i}
\end{aligned}
$$

where $\sigma_{\text {sum }}$ is the sum of equation (10):

$\sigma_{\text {sum }}=\sum_{i \in \mathbb{N}_{\sigma}} v_{i} \sigma_{i}^{p}$

and the vector $\boldsymbol{\lambda}_{i}^{\sigma}$ contains the $i$ 'th element's degrees of freedom of the adjoint vector for the stress constraint. This adjoint field, $\boldsymbol{\lambda}^{\sigma}$, is calculated from the following adjoint problem:

$\mathbf{K} \boldsymbol{\lambda}^{\sigma}=-\frac{\partial f_{s}^{T}}{\partial \mathbf{u}}$

where the right-hand side is obtained through the finite element assembly of the element contributions:

$\frac{\partial f_{s}{ }^{T}}{\partial \mathbf{u}_{i}}=\frac{c \sigma_{s u m}{ }^{\frac{1}{p}-1} v_{i} \sigma_{i}^{p-1} \tilde{\tilde{\rho}}_{i}^{q}}{\sigma_{v m, i}} \mathbf{B}^{T} \mathbf{C}_{0} \mathbf{V} \boldsymbol{\sigma}_{i}$

\subsubsection{Constant beta vs. beta continuation}

As stated earlier, the value of $\beta$ determines the slope of the smoothed Heaviside function, described in equation (4). Generally, $\beta$ is initialized with a small value, for instance 1 , and then gradually increased using a continuation procedure. This can cause the algorithm to be very slow in convergence, besides the appearance of oscillatory and instable behavior when $\beta$ is increased.

As mentioned in section 2.2 , the relation between stress level and density is very non-linear. This is very pronounced when updating values for $\beta$. Especially for large values, the procedure of updating can cause perturbations that lead the algorithm to loose the path to convergence.

Recently, Guest et al. (2011) proposed a modification of the initial optimization settings in MMA, in order to allow the algorithm to start with large values for

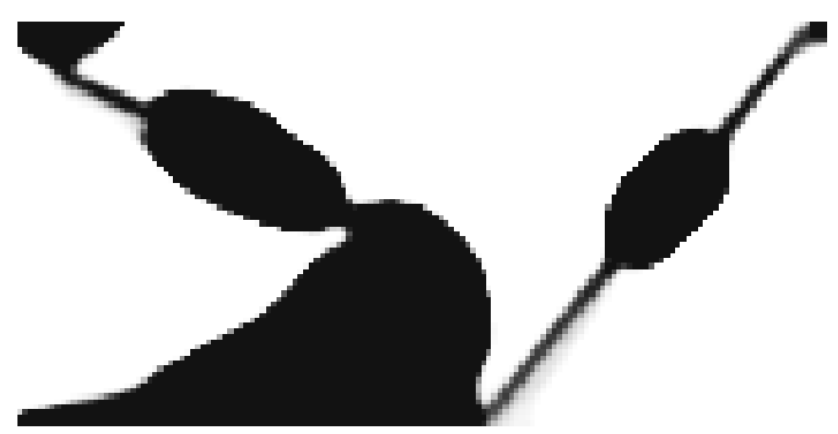

(a) constant $\beta$ : $f=-3.95, \sigma^{*}=9, M_{n d}=3.3 \%$

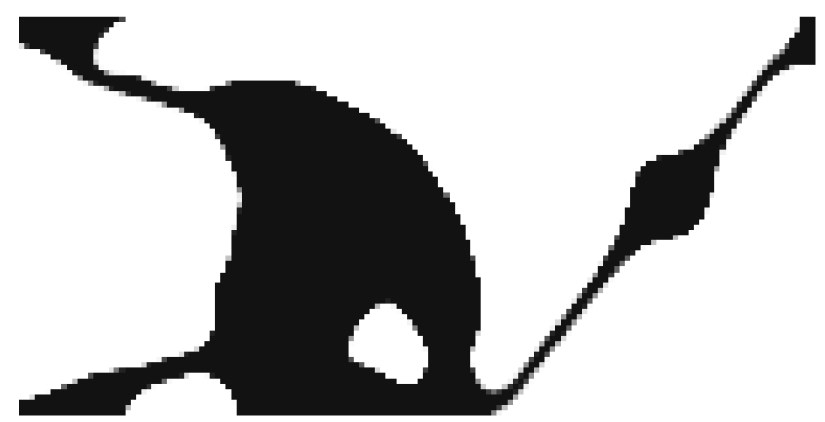

(b) $\beta$ continuation: $f=-3.99, \sigma^{*}=9, M_{n d}=1.2 \%$

Fig. 3 Convergence problems using a constant value for $\beta$. Without the $\beta$-continuation strategy, pronounced gray regions are seen in the hinge regions (a).

$\beta$. A tightening of the MMA asymptotes tells the algorithm to use a very conservative approximation from the onset of optimization. The initial asymptotes are made dependent of $\beta$ and this alternative to the continuation method seems to be a good way in preventing perturbations and slow convergence.

However, for the formulation presented in this work, starting with a large value for $\beta$ caused an additional problem. Mainly when dealing with very low thresholds for stress, the algorithm seems to find particularly poor local minima. Fig. 3 shows an example where the algorithm converged to a mechanism with a pronounced gray area in the hinge regions. This problem did not appear when a continuation procedure was applied.

To avoid this problem and overcome the perturbation issue, this work uses a $\beta$-continuation procedure alongside the tightening of the asymptotes proposed by Guest et al. (2011) every time the curve slope is updated. This procedure slows the convergence, but allows the algorithm to both control the stresses and in the authors' experience generally produces better optimized designs. 


\section{Numerical examples}

In compliant mechanism design, there are several benchmark problems widely used to verify if a given formulation converges towards a hinge-free design. These benchmark tests are prone to present hinges if the formulation does not help to prevent them. In the following we use the benchmark examples as e.g. used by Sigmund (2009), but modified to use physical dimensions and material properties (Sigmund, 1997) in order to ensure a physically reasonable meaning of the stress constraint.

\subsection{Compliant inverter}

The first example is the compliant inverter, where the output port is expected to move in the opposite direction compared to the input port (Fig. 4). The dark gray squares in the upper left and right corners are a group of elements that are inactive; that is they are forced to have density equal to 1 and are ignored in the calculations of the global stress measure, i.e. excluded from the set $\mathbb{N}_{\sigma}$. Due to the point load nature of the input and output points, these areas will inherently exhibit large stresses which would overshadow other highly stressed regions if not excluded from the design domain and the stress norm calculation. In reality, the input actuator and workpiece have finite contact regions with the compliant mechanism, in turn avoiding this stress singularity issue.

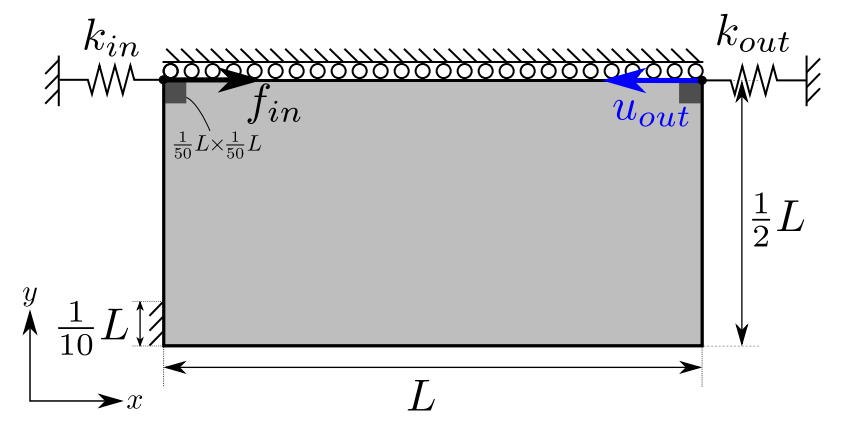

Fig. 4 Sketch of the compliant inverter problem setup.

Silicon is chosen as the base material with Young's moduli for the solid and void phases as $E_{0}=180$ [GPa] and $E_{\min }=10^{-9} E_{0}$, respectively, and the Poisson ratio is $\nu=0.3$. The force applied at the input port is $f_{i n}=1000[\mu N]$, the input spring stiffness is $k_{i n}=$ $2 \times 10^{-4}\left[\frac{N}{\mu \mathrm{m}}\right]$, and the output spring stiffness is $k_{\text {out }}=$ $2.5 \times 10^{-4}\left[\frac{N}{\mu \mathrm{m}}\right]$. The domain length is set to $L=$ $150[\mu \mathrm{m}]$ and the thickness is set $7[\mu \mathrm{m}]$. The penalization parameter (SIMP) is $k=3$ and the relaxation parameter for stress is set to $q=1$ until convergence, then it is updated to 0.5 to further reduce the gray areas for all presented examples. In the work by Le et al. (2010), a fixed value of $q=0.5$ was used from the start, however, for mechanism design with a stress constraint we found that the most stable behavior is obtained with the described two-step approach for this parameter. The volume fraction is $V^{*}=0.3$ and the stress limit, $\sigma^{*}$, will be stated for each result. For all examples, a density filter radius of $\frac{5.6 L}{100}[\mu \mathrm{m}]$ is used, $\eta=0.5$ and $\beta$ is doubled every 100 iterations until $\beta_{\max }=150$ from an initial value of 1 , except for cases shown in section 3.1.1 where the maximum value for $\beta$ are specified.

In the following we investigate the influence of mesh refinement, stress constraint value and output stiffness value for the inverter example.

\subsubsection{Mesh refinement study}

In order to investigate whether the imposed stress constraint results in mesh-independence, a mesh refinement study is carried out.

Fig. 5 shows results for several meshes, both with and without an active stress constraint, using the same parameters, except for $\beta_{\max }$. This value is set to 50 , $100,150,200$ and 300 for the meshes $50 \times 25,100 \times 50$, $150 \times 75,200 \times 100$ and $300 \times 150$, respectively, due to the fact that a higher $\beta$ value is needed to ensure the same level of gray-scale when the filter radius is larger relative to the element size (Guest et al., 2011). Stress levels in the optimized mechanisms are shown using the same gray scale for all designs with black meaning high stress and white meaning zero stress.

It can be seen that the topologies are qualitatively the same, with small variations. The most significant change is the length of the compliant members replacing the one-node-connected hinges for the stress constrained designs. It is seen that the length of these compliant members decreases with mesh-refinement. Fig. 6 shows close-ups of the hinge regions for the three finest meshes. Here it can be observed that the stress constraint effectively removes the one-node-connected hinges and replaces them with a compliant member. It is also observed that the thickness and length of the compliant member varies with mesh-refinement. To avoid stress concentrations the optimizer replaces the onenode-connected hinge with a compliant member with minimal bending resistance that satisfies the stress constraint. As the mesh is refined, the member gets thinner meaning that the member can be shorter and still ensure the same small bending stiffness and stress level as for the coarser design. Obviously, the stress distribu- 


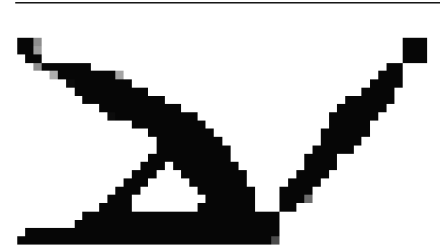

(a) $50 \times 25, f=-4.136$

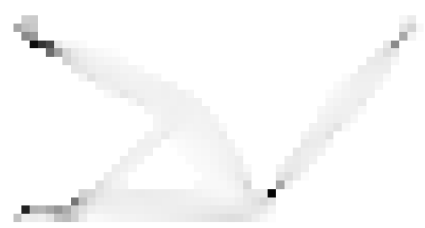

(b) $\sigma^{*}=14, \sigma_{\max }=14$

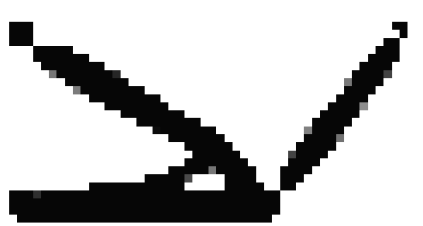

(c) $50 \times 25, f=-4.425$

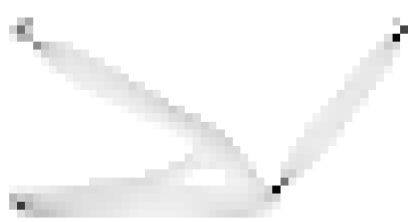

(d) $\sigma_{\max }=34.9$

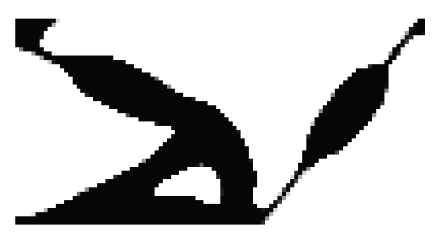

(e) $100 \times 50, f=-4.147$

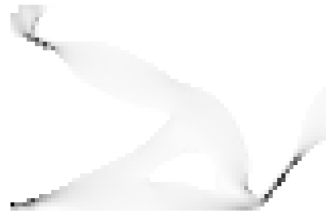

(f) $\sigma^{*}=14, \sigma_{\max }=14$

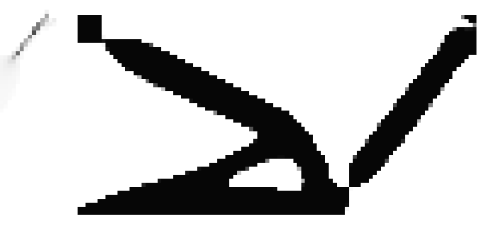

(g) $100 \times 50, f=-4.311$

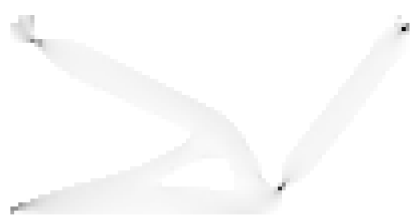

(h) $\sigma_{\max }=26.8$

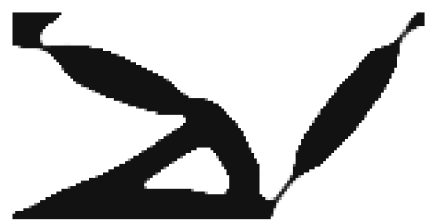

(i) $150 \times 75, f=-4.235$

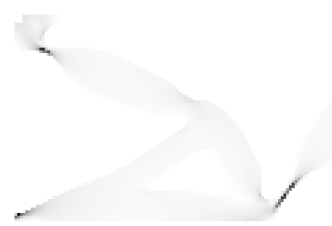

(j) $\sigma^{*}=14, \sigma_{\max }=14$

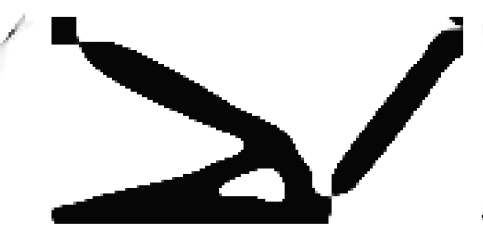

(k) $150 \times 75, f=-4.321$ (l) $\sigma_{\max }=25.4$

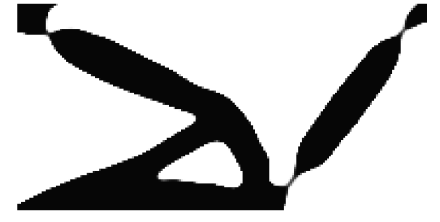

(m) $200 \times 100, f=-4.285$

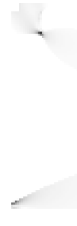

(n) $\sigma^{*}=14, \sigma_{\max }=14$

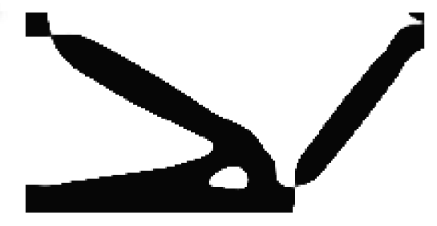

(o) $200 \times 100, f=-4.320$ (p) $\sigma_{\max }=24.1$

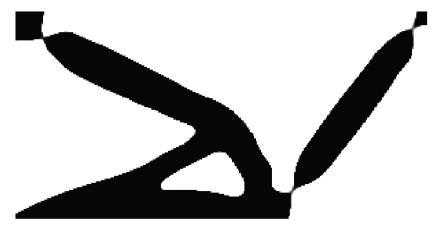

(q) $300 \times 150, f=-4.299$ (r) $\sigma^{*}=14, \sigma_{\max }=14$

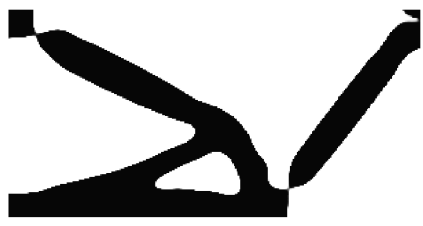

(s) $300 \times 150, f=-4.321$ (t) $\sigma_{\max }=18.9$

Fig. 5 Inverter compliant mechanism with (left) and without (right) stress constraints for different meshes. The units for all examples are in $\mu \mathrm{m}$ for $f$ and GPa for $\sigma^{*}$ and $\sigma_{\max }$.

tion in the compliant member is not well-modeled by only two element discretizing its cross-section. Also, the hinges for the finer meshes do show a bit of grey scale (see Sec. 3.1.2 for a discussion of this issue). Nevertheless, this example shows that adding a stress constraint by itself does not ensure mesh-independent designs although it does eliminate the one-node-connected hinge issue. It appears that the addition of the stress constraint to compliant mechanism design still must be accompanied by a minimum feature size constraint in order to ensure accurate modeling and optimization for compliant mechanism design.

It is very interesting to note that despite a significantly reduced maximum stress, the performance is not remarkably lower for the stress constrained designs compared to the non-constrained ones. This aspect will be further investigated in the following subsection.

\subsubsection{Effect of stress constraint and Pareto optimality}

In order to further investigate the effect of imposing a stress constraint on the performance of the optimized designs, a Pareto front is sought by optimizing the compliant inverter for several stress limits of decreasing value. In all examples a mesh of $150 \times 75$ is applied.

The Pareto plots in Fig. 8 (and later in Fig. 13) show two sets of results. "Optimized design" denotes the results as obtained in the optimization process and "thresholded design" denotes their subsequent solid and void interpretations. Here, intermediate densities are 

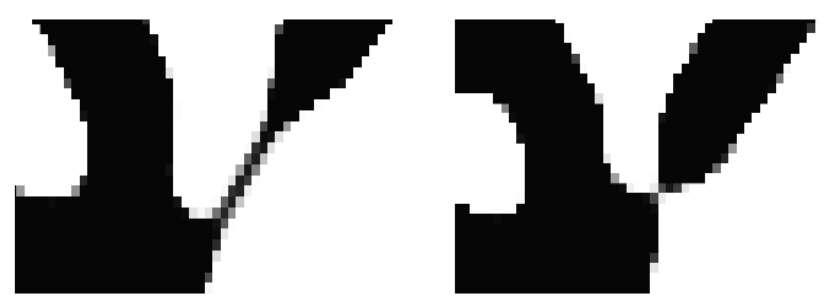

(a) $150 \times 75$
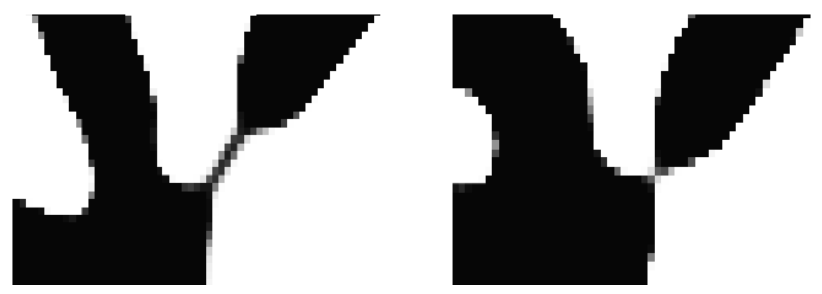

(b) $200 \times 100$
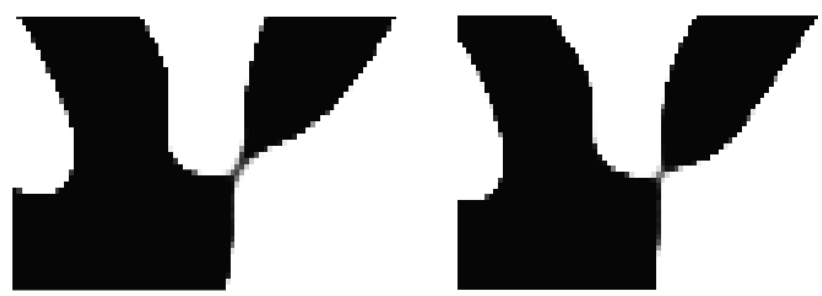

(c) $300 \times 150$

Fig. 6 Close-ups of the hinge region of a selection of results presented in Fig. 5. Stress constrained left and no constraint right.

mapped to 0 or 1 using a sharp threshold value of 0.5 . Fig. 7 shows an example of an optimized inverter before and after thresholding. One can notice from the Pareto plots that the $0-1$ interpretation causes a small performance decrease in all examples. This is partly due to the actual density change that causes changed stiffness and hence changed response but also due to the stress evaluation itself that dependents on density. The discrepancies in compliance stay below a few percent and below 10-20\% for the stress evaluation with a decreasing tendency with mesh refinement. We observe that the proper choice of penalization parameters $k$ and $q$ alone is not enough to ensure completely $0-1$ designs because the optimizer always will take advantage of the possibility of reducing stress by slightly lowering the density. However, the error observed after thresholding are small enough to support the conclusions that we draw in this paper.

Fig. 8 shows the Pareto front obtained from optimizing the compliant inverter for several stress limits. The $x$-axis is the inverse of the absolute displacement at the output port, $\frac{1}{\left|u_{\text {out }}\right|}$, and the $y$-axis is the stress (relaxed $p$-norm (i.e. $\tilde{\sigma}_{P N}$ ) for the grey scale design and $\sigma_{\max }$ for the thresholded design).

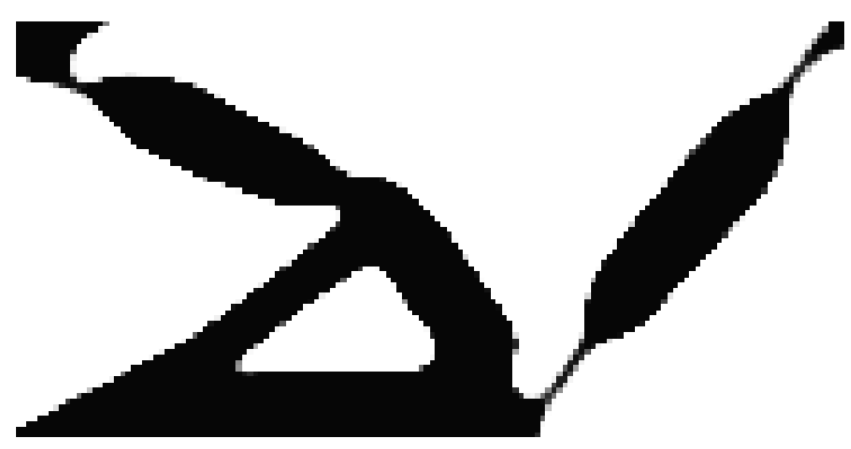

(a) $f=-4.235, \sigma^{*}=14, \sigma_{\max }=13.99$

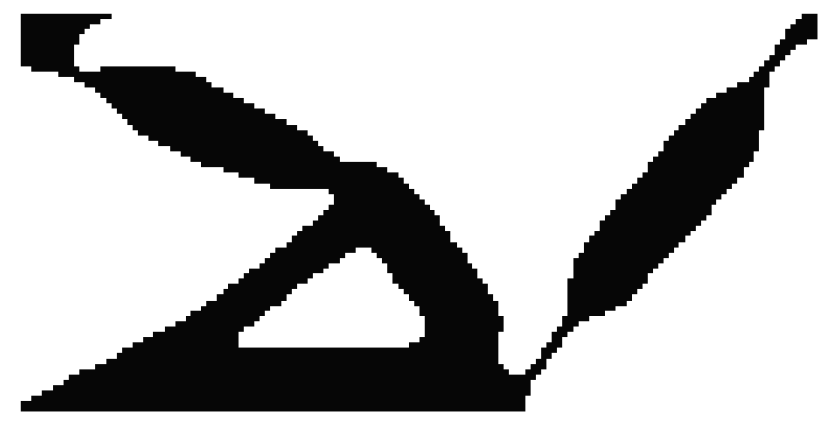

(b) $f=-4.225, \sigma_{\max }=15.12$

Fig. 7 Optimized topology (a) and the respective thresholded design (b) for the inverter mechanism. All the values for $f$ and $\sigma$ are in $\mu \mathrm{m}$ and GPa, respectively.

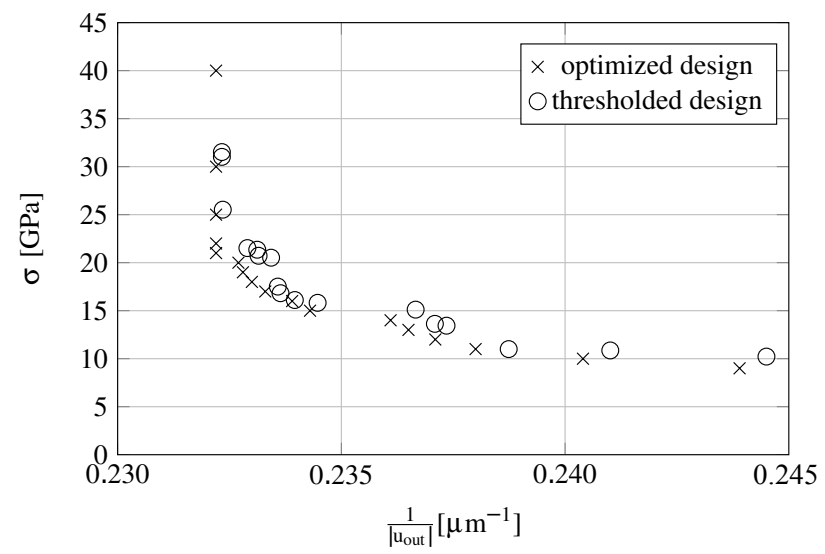

Fig. 8 Pareto curve for the compliant inverter corresponding to the results in Fig. 9.

The obtained points show a clear trade-off between the stress level and the performance of the inverter. However, it is very interesting to note, that quite a low stress limit can be imposed before detrimental effects are observed on the mechanism performance. For example, it is possible to lower the stress level from 40 to 15 and only loosing slightly more than $1 \%$ in output displacement. As will be discussed in more detail in the following, the mechanisms change from lumped compli- 


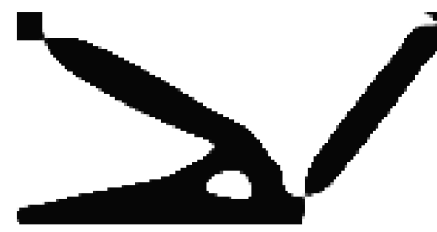

(a) $f=-4.331, \sigma^{*}=40$

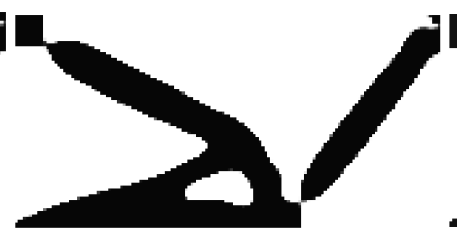

(b) $f=-4.300, \sigma^{*}=30$

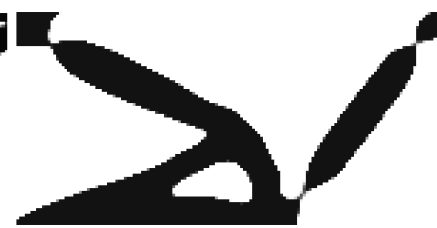

(c) $f=-4.305, \sigma^{*}=22$

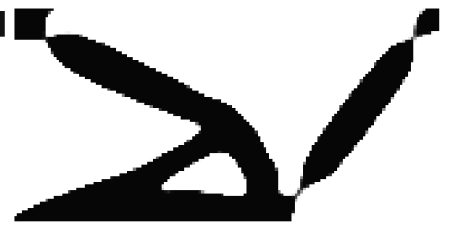

(d) $f=-4.305, \sigma^{*}=21$

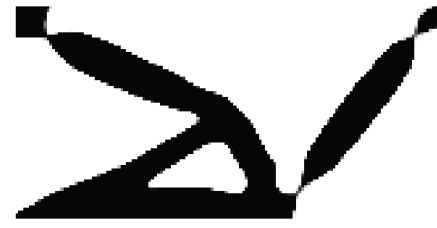

(e) $f=-4.293, \sigma^{*}=20$

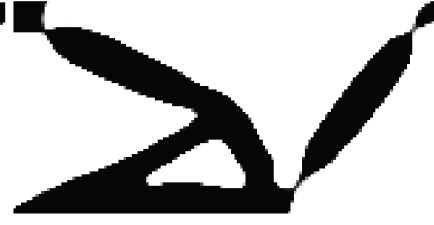

(f) $f=-4.287, \sigma^{*}=19$

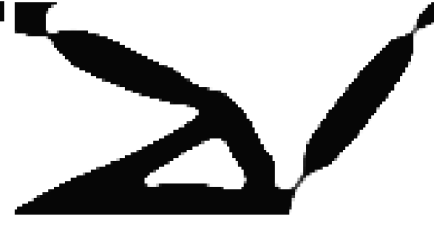

(g) $f=-4.283, \sigma^{*}=18$

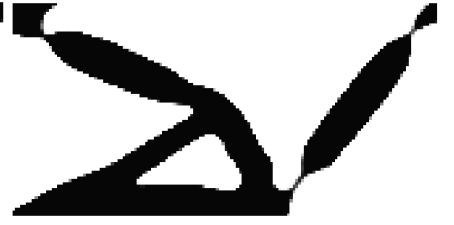

(h) $f=-4.286, \sigma^{*}=17$

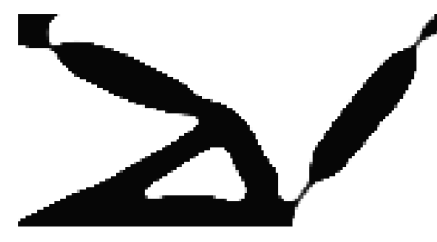

(i) $f=-4.275, \sigma^{*}=16$

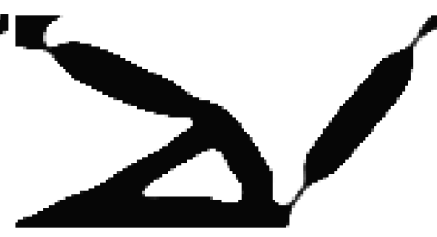

(j) $f=-4.267, \sigma^{*}=15$

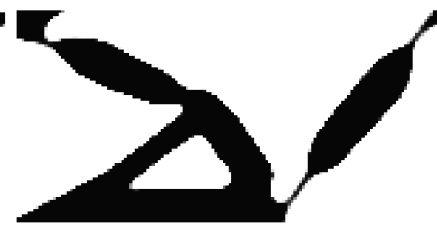

(k) $f=-4.235, \sigma^{*}=14$

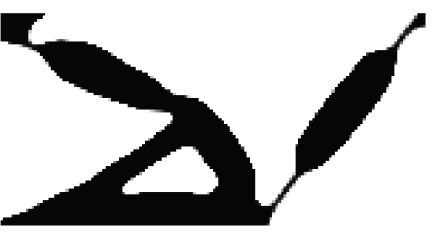

(l) $f=-4.227, \sigma^{*}=13$

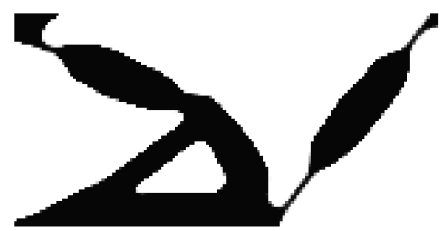

(m) $f=-4.217, \sigma^{*}=12$

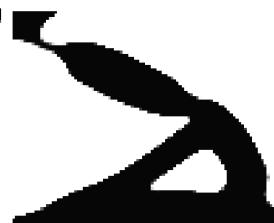

(n) $f=-4.201, \sigma^{*}=11$

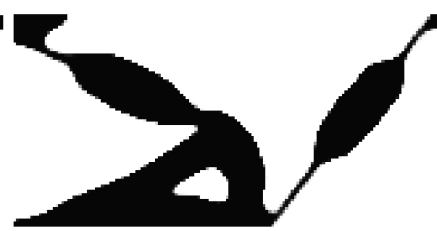

(o) $f=-4.159, \sigma^{*}=10$

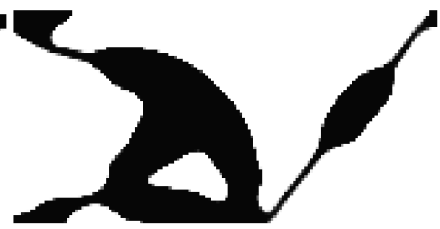

(p) $f=-4.100, \sigma^{*}=9$

Fig. 9 Optimized topologies for the compliant inverter problem with several stress constraint limits. All the values for $f$ and $\sigma^{*}$ are in $\mu \mathrm{m}$ and $\mathrm{GPa}$, respectively.

ant to distributed compliant as the stress constraint is lowered.

This is further emphasized by Fig. 9, which shows the same optimized topologies for various levels of the stress limit. The examples vary from a high stress limit, $\sigma^{*}=40$ (Fig. 9(a)), where the stress constraint is not active, to a very low value, $\sigma^{*}=9($ Fig. $9(\mathrm{p}))$, where it can be seen that the topology is significantly different and exhibits no local hinges. It is observed that as the stress limit is decreased, the length of the compliant members is continually increased in order to distribute the deformation and lower the stresses to comply with the constraint. At the same time the compliant members become thicker in order to accommodate the increasing demand on longitudinal stiffness as the members become longer. We note here that for all examples the volume constraint $f_{v}$ is active.

Fig. 10 shows the stress distributions for the optimized topologies from Fig. 9. For this case the scales are different for each subfigure but as earlier, black de- notes high stress and white low stress. It can be seen that as the stress limit is lowered, the optimized designs are more homogeneously stressed and it is clear to see that mechanisms transit from being lumped to being distributed.

The ultimate strength for silicon is not well-defined. Silicon is a brittle material at room temperature and the fracture strength depends on many factors such as length-scale, surface roughness, environment, geometry, crystal-orientation, and so forth. Cook (2006) lists many different values mostly within the range of 1-18 $\mathrm{GPa}$ which corresponds to the interval of stress limits used in the examples here.

We note that Pareto plot in Fig. 8 is not very smooth and that there are points which are clearly non-optimal. Clearly, one could produce better and smoother Pareto curves by using neighbor points as starting guesses and improved continuation strategies. Here we chose simply to plot the raw points resulting from individual standardized runs showing that the designs problems 
(a) $f=-4.331, \sigma^{*}=40$

(b) $f=-4.300, \sigma^{*}=30$

(c) $f=-4.305, \sigma^{*}=22$

(d) $f=-4.305, \sigma^{*}=21$

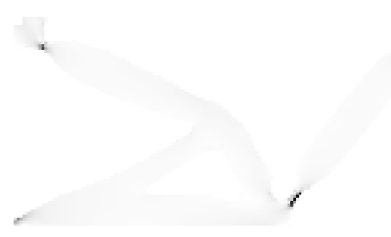

(e) $f=-4.293, \sigma^{*}=20$

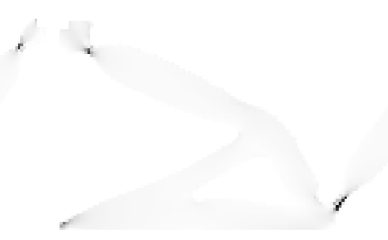

(f) $f=-4.287, \sigma^{*}=19$

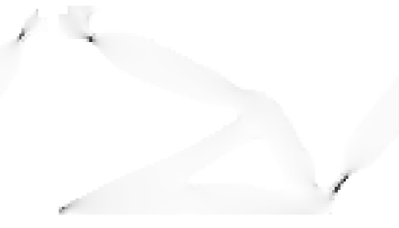

(g) $f=-4.283, \sigma^{*}=18$

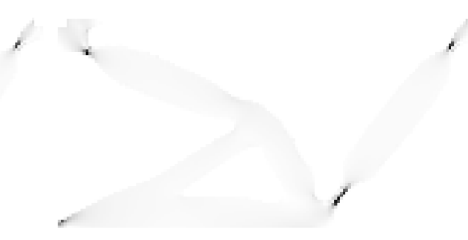

(h) $f=-4.286, \sigma^{*}=17$
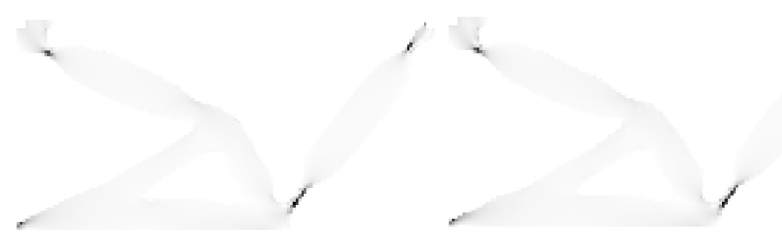

(i) $f=-4.275, \sigma^{*}=16$

(j) $f=-4.267, \sigma^{*}=15$

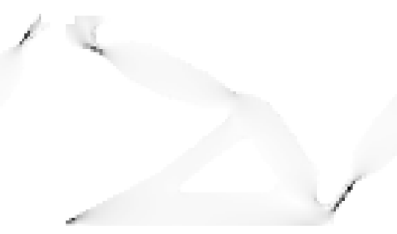

(k) $f=-4.235, \sigma^{*}=14$

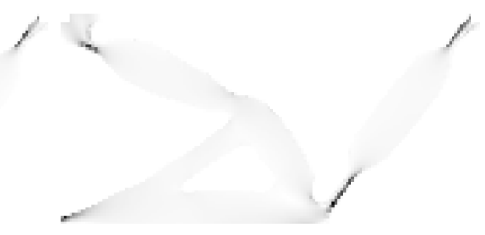

(l) $f=-4.227, \sigma^{*}=13$

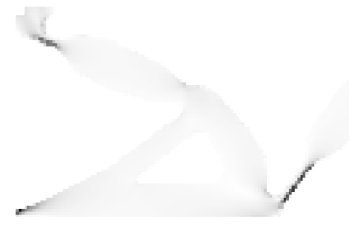

(m) $f=-4.217, \sigma^{*}=12$

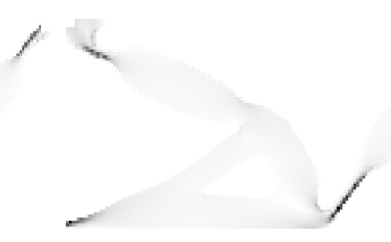

(n) $f=-4.201, \sigma^{*}=11$

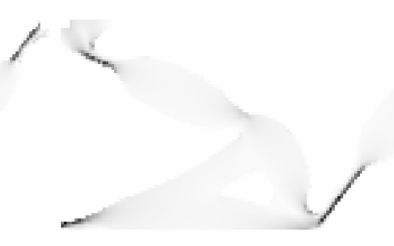

(o) $f=-4.159, \sigma^{*}=10$

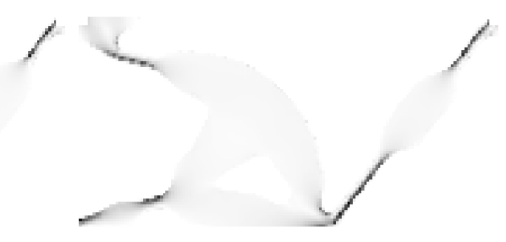

(p) $f=-4.100, \sigma^{*}=9$

Fig. 10 Stress distributions for the optimized topologies shown in Fig. 9 for the compliant inverter problem. All the values for $f$ and $\sigma^{*}$ are in $\mu \mathrm{m}$ and GPa, respectively.

are highly complex and that slight change of optimization parameters may convergence to different local minima. Nevertheless, the resulting Pareto curves clearly prove the tendencies and conclusions discussed above and later for the gripper example.

\subsubsection{Effect of output stiffness}

A mechanism designed to work with a high output stiffness is expected to deliver a small output displacement but large force and vice versa. This relationship is expected for both compliant and rigid-body mechanisms. To be able to deliver a large force for a high output spring stiffness, the optimized mechanism will seldomly exhibit hinge regions. Therefore, specifying a large output force or small input displacement indirectly removes lumped hinges but on the other hand results in mechanism with limited output kinematics. Hence many of the optimization formulations including global stiffness measures seen in the literature indeed avoid the lumped hinge issue, but at the same time the resulting mechanisms have very limited output motions. The inclusion of a stress constraint does not directly imply high global stiffness and hence stress constrained mechanisms are expected to deliver larger output displacements.

Fig. 11 shows results for different output springs, $k_{\text {out }}=2.5 \times\left\{10^{-2}, 10^{-3}, 10^{-4}, 10^{-5}\right\}$, both without and with a stress limit of $\sigma^{*}=14$. It can be seen that the stress-constrained formulation ensures that one-nodeconnected hinges do not form for any of the output stiffnesses. It is interesting to note that the thickness and length of the compliant members vary quite significantly when the output spring stiffness is varied; an increase in the output spring stiffness leads to an increased thickness and a decreased length of the compliant members. It is also interesting to note that the difference in the optimized material distributions, between the stress-constrained and the unconstrained cases increases as the output spring stiffness is decreased. For low output spring stiffnesses the requirement to low stiffness (e.g. one-node-connected) hinges increases. If 

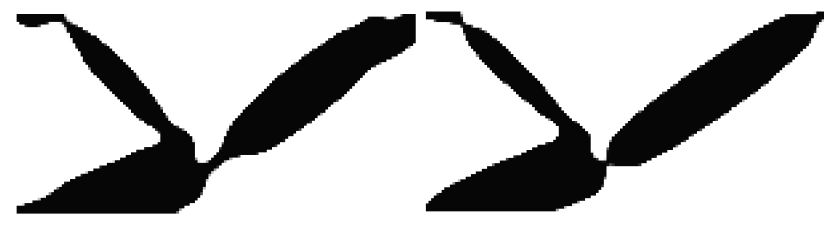

\begin{abstract}
(a) $f=-0.197$, $k_{\text {out }}=2.5 \times 10^{-2}$, $M_{n d}=0.756 \%$
\end{abstract}

(b) $f=-0.219$, $k_{\text {out }}=2.5 \times 10^{-2}$, $\sigma_{\max }=37.6$ $M_{n d}=0.505 \%$

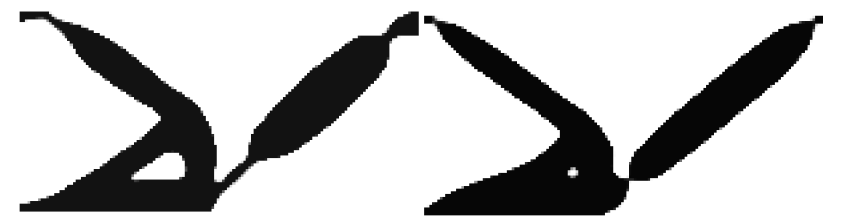

(c) $f=-1.132$, $k_{\text {out }}=2.5 \times 10^{-3}$, $M_{n d}=0.763 \%$

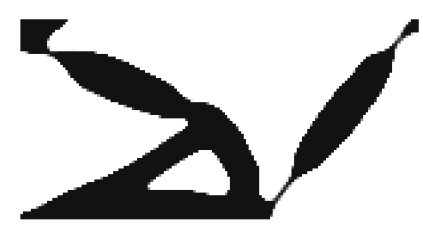

(e) $f=-4.235$, $k_{\text {out }}=2.5 \times 10^{-4}$, $M_{n d}=0.793 \%$

$$
\begin{aligned}
& \text { (d) } f=-1.204, \\
& k_{\text {out }}=2.5 \times 10^{-3}, \\
& \sigma_{\text {max }}=30.3, \\
& M_{n d}=0.587 \%
\end{aligned}
$$

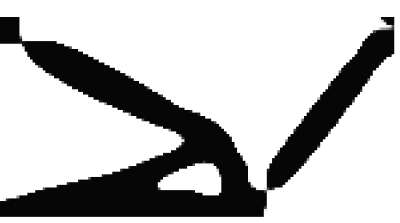

$$
\begin{aligned}
& \text { (f) } f=-4.321, \\
& k_{\text {out }}=2.5 \times 10^{-4} \text {, } \\
& \sigma_{\text {max }}=25.4, \\
& M_{n d}=0.520 \%
\end{aligned}
$$

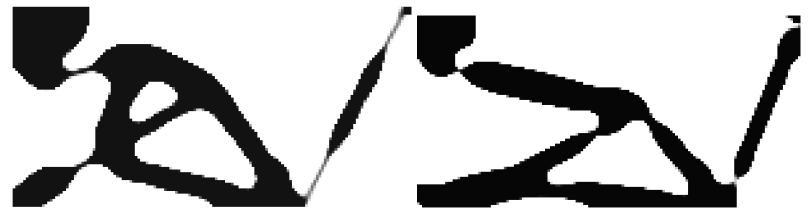

$$
\begin{aligned}
& \text { (g) } f=-13.344, \\
& k_{\text {out }}=2.5 \times 10^{-5}, \\
& M_{n d}=0.988 \%
\end{aligned}
$$

$$
\begin{aligned}
& \text { (h) } f=-13.800, \\
& k_{\text {out }}=2.5 \times 10^{-5} \text {, } \\
& \sigma_{\text {max }}=61.5, \\
& M_{n d}=0.520 \%
\end{aligned}
$$

Fig. 11 Inverter compliant mechanism with and without stress constraint for several workpiece output stiffnesses. In examples where stress is active (left side), $\sigma^{*}=14$.

this is not possible due to the stress constraint, the only alternative is to make very long and slender hinges that minimize the bending resistance. If there is still a longitudinal force requirement, the compliant member thickness will increase in turn requiring an even longer compliant member (or a fully distributed compliant mechanism) to obtain the required low bending stiffness.

As commonly observed for the unconstrained case, the angle of the right-most member changes to accommodate the different load transfer conditions (Sigmund,
2001) for varying output stiffness. In this regard, it is interesting to see that the optimized angles are very similar for the stress-constrained and unconstrained designs. Obviously these output angles will change when the mechanisms will be analyzed and optimized based on finite deformation theory in future works.

\subsection{Compliant gripper}

The compliant gripper benchmark problem is used as the second example. It has a similar domain as the inverter, with a small difference in the region where the workpiece is defined to be. In order to guarantee room for the workpiece, a large void region is specified and a solid region is defined under the void region, to ensure the presence of jaws, c.f. Fig. 12.

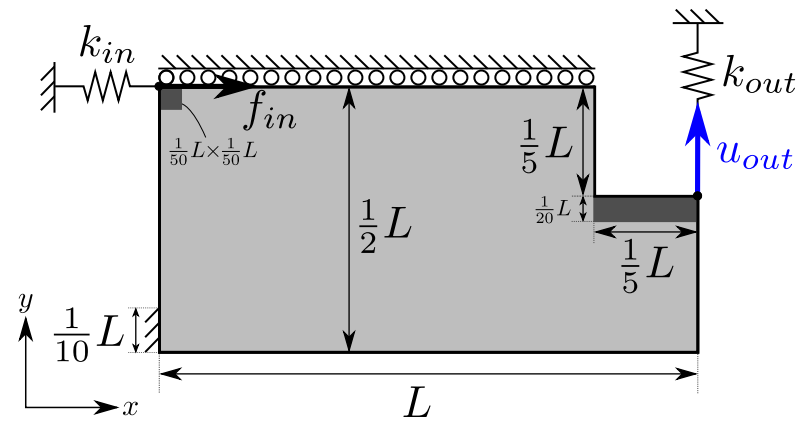

Fig. 12 Sketch of the problem setup for the compliant gripper.

As for the compliant inverter, the domain represents only half of the mechanism. The dark gray square in the upper left corner follows the same idea as in the inverter case. The solid region defining the jaws is also excluded from the stress constraint.

The mechanical properties of silicon are the same as for the previous example. The force applied at the input port is $f_{\text {in }}=1000[\mu N]$, the input spring stiffness is $k_{i n}=5 \times 10^{-4}\left[\frac{N}{\mu \mathrm{m}}\right]$ and the output spring stiffness is $k_{\text {out }}=1.0 \times 10^{-3}\left[\frac{N}{\mu \mathrm{m}}\right]$. The domain length is $L=150[\mu \mathrm{m}]$ and the thickness is $7[\mu \mathrm{m}]$. The allowed volume fraction is $V^{*}=0.25$ and the stress limit, $\sigma^{*}$, varies as specified. Other parameters are as previously specified in section 3.1 .

Fig. 13 shows the Pareto curve obtained for the compliant gripper problem and again a clear trade-off can be observed between the stress levels and the mechanism performance. As for the compliant inverter, it is seen that a quite significant limit on the stress can be imposed without leading to a significant decrease in the output displacement. 

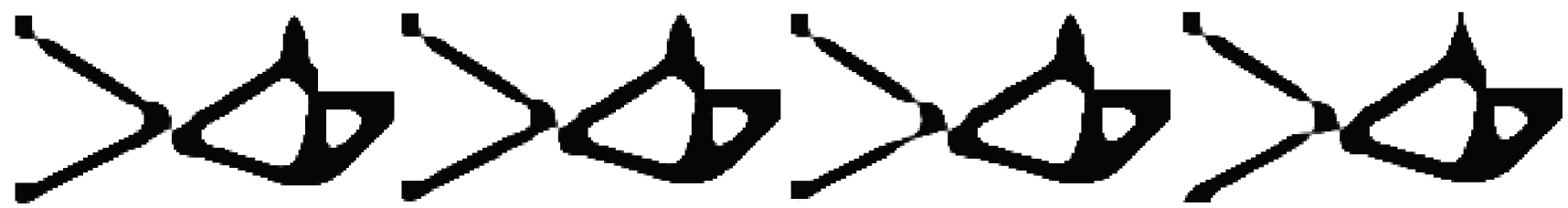

(a) $f=0.586, \sigma^{*}=10$,

$f_{v}=0.25$ (b) 0.25

(d) $f=0.57$
$f_{v}=0.25$
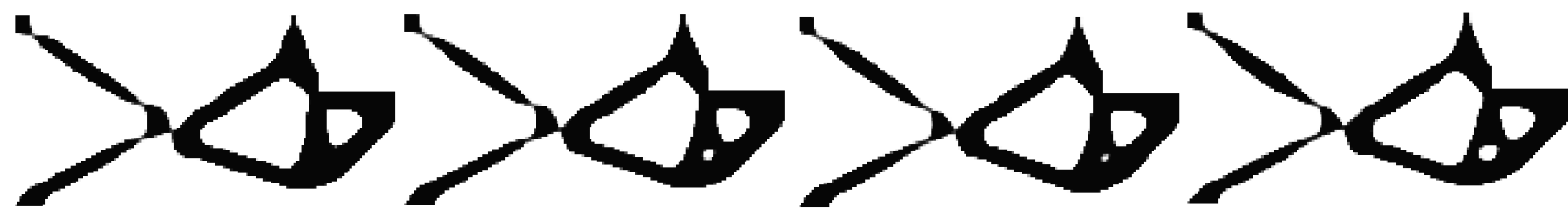

(e) $f=0.568, \sigma^{*}=6, f_{v}=(\mathrm{f}) f=0.566, \sigma^{*}=5, f_{v}=0.25(\mathrm{~g}) f=0.561, \sigma^{*}=4.8$,

(h) $f=0.559, \sigma^{*}=4.6$, 0.25 $f_{v}=0.25$

$f_{v}=0.25$
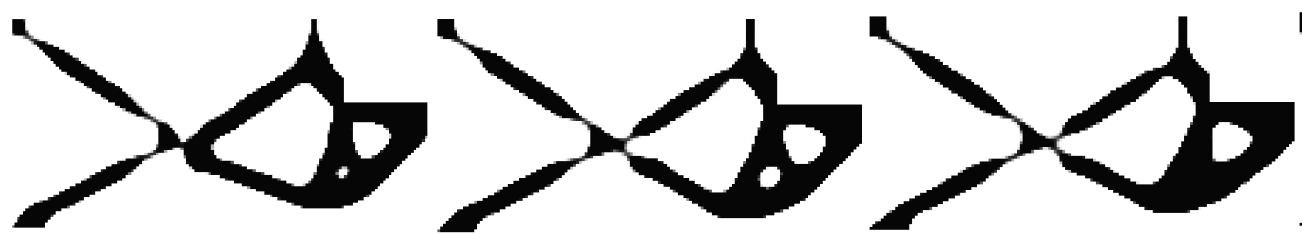

(i) $f=0.558, \sigma^{*}=4.4$,

(j) $f=0.548, \sigma^{*}=4.2$,

(k) $f=0.532, \sigma^{*}=4$,

$f_{v}=0.25$

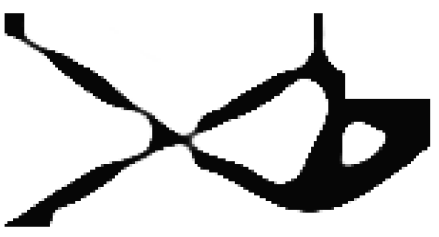

$f_{v}=0.25$

$f_{v}=0.25$

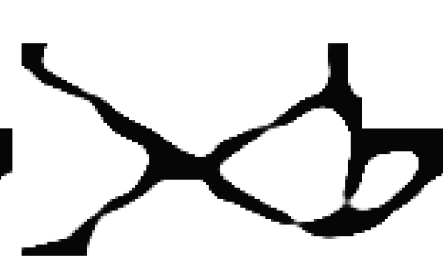

(l) $f=0.513, \sigma^{*}=3.8$,

$f_{v}=0.25$
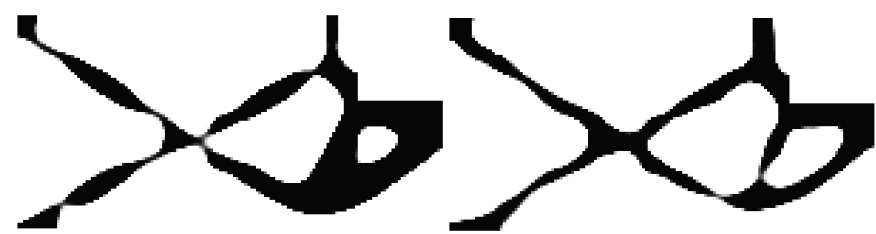

(m) $f=0.499, \sigma^{*}=3.6$,

(n) $f=0.442, \sigma^{*}=3.4$,

(o) $f=0.431, \sigma^{*}=3.2$,

$f_{v}=0.23$

$f_{v}=0.20$

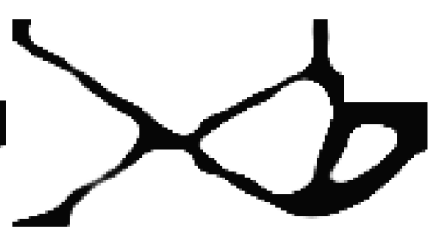

(p) $f=0.420, \sigma^{*}=3$

$f_{v}=0.21$

Fig. 14 Optimized topologies for the compliant gripper problem with various stress constraint limits. All the values for $f$ and $\sigma^{*}$ are in $\mu \mathrm{m}$ and GPa, respectively.

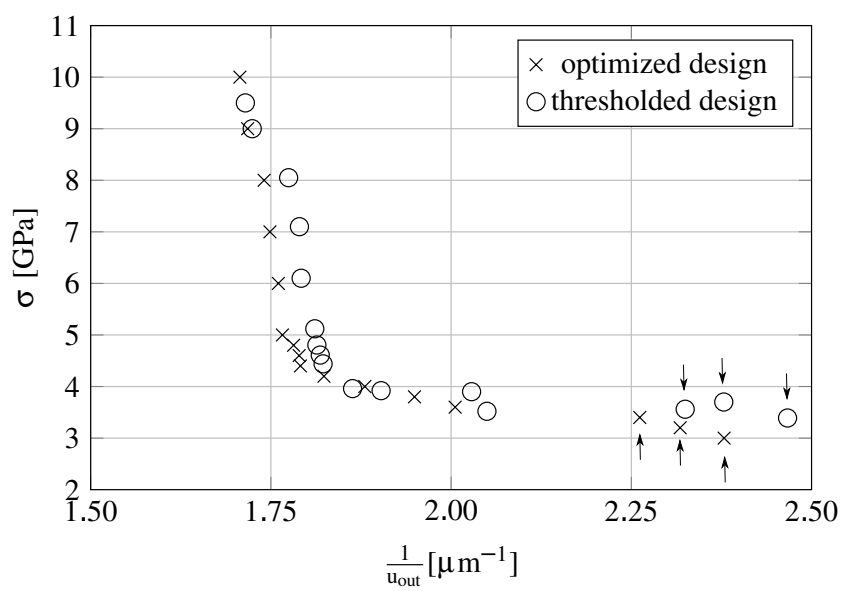

Fig. 13 Pareto curve for the compliant gripper problem (the arrows indicate designs where the volume constraint is not active).
Fig. 14 shows several optimized topologies for various levels of the stress limit. The examples vary from a stress limit of $\sigma^{*}=10$ (Fig. 14(a)) to a very low value, $\sigma^{*}=3$ (Fig. 14(p)). As for the compliant inverter problem, it is observed that as the stress limit is decreased, the presence of local hinges is diminished. However, it appears that the stress limit must be quite a bit lower in order to push the optimized design towards distributed hinges. It is important to note that for very low stress limits, the volume constraint $f_{v}$ is not active for the final designs.

Fig. 15 shows the stress distributions for the optimized topologies presented in Fig. 14. It can be seen that when the stress limit is very low, the optimized designs hardly exhibit local stress concentrations. Instead they become truly distributed compliant mechanisms with a relatively homogeneous stress distribution. In 


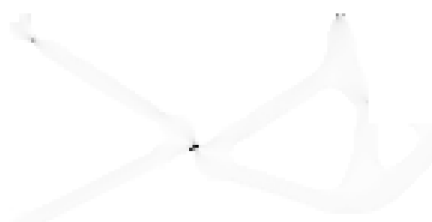

(a) $f=0.586, \sigma^{*}=10$, $f_{v}=0.25$

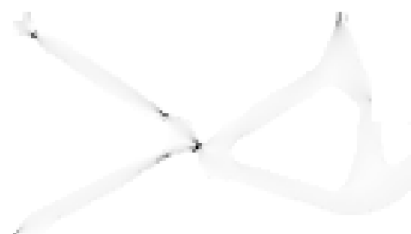

(e) $f=0.568, \sigma^{*}=6$, $f_{v}=0.25$

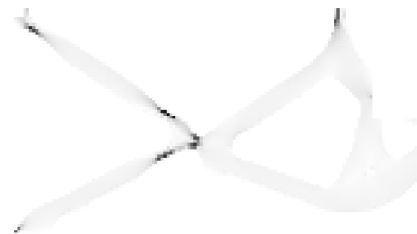

(i) $f=0.558, \sigma^{*}=4.4$, $f_{v}=0.25$

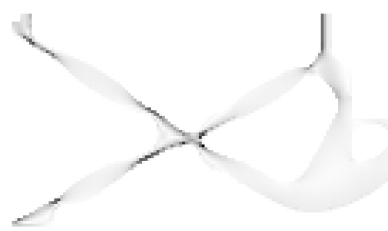

(m) $f=0.499, \sigma^{*}=3.6$,

$f_{v}=0.25$

Fig. 15 Stress distributions for the optimized topologies shown in Fig. 14 for the compliant gripper problem. All the values for $f$ and $\sigma^{*}$ are in $\mu \mathrm{m}$ and GPa, respectively.

the limit of very small stress limit, it is noted that the volume constraint becomes inactive. In order to satisfy the stress constraint, the resulting mechanism will have very thin and flexible members and can not make use of the superfluous material.

Fig. 16 shows a sample of the thresholded design obtained for $\sigma^{*}=4.4$. All conclusions drawn for the inverter example are also valid here. In this example an increase of around $1.1 \%$ is observed for the maximum stress following thresholding.

\section{Discussion and conclusions}

In this paper we have added a stress constraint to the classical compliant mechanism design problem. The stress constraint is shown to have a strong influence on the resulting designs. The usual issue of one-node-connected and highly stressed hinges is eliminated. The lumped hinges are replaced with compliant members. As the stress level is lowered or the output stiffness is reduced, the optimized compliant mechanisms go from being lumped to being distributed. Hence, we conclude that there is not a clear answer to the long standing question: are optimal compliant mechanisms based on lumped or distributed compliance? The answer depends on material properties, stress constraint, input and output stiffnesses as well as design domain. However, it appears that the proposed algorithm can be used to answer the question for given design conditions.

Another important question answered in the paper is if the imposing of a stress constraint would yield a 


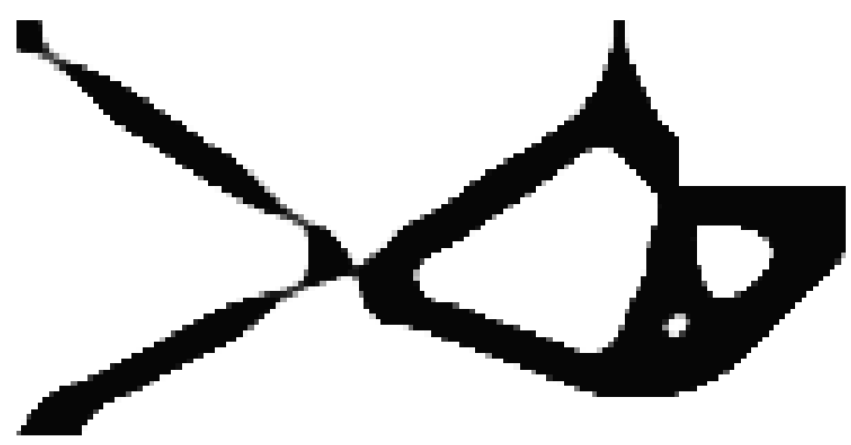

(a) $f=0.558, \sigma^{*}=4.40, \sigma_{\max }=4.39$

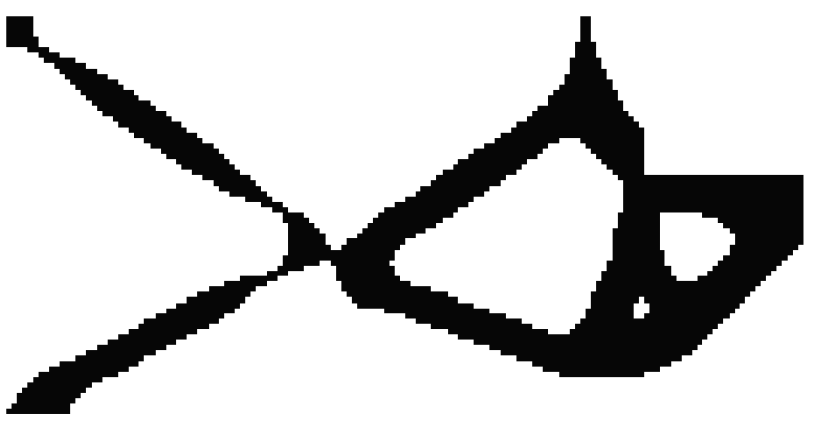

(b) $f=0.549, \sigma_{\max }=4.45$

Fig. 16 Optimized topology (a) and the respective thresholded (b) design for the gripper. All the values for $f$ and $\sigma$ are in $\mu \mathrm{m}$ and $\mathrm{GPa}$, respectively.

mesh-independent solution? The obtained results indicate that this is not the case. The stress constraint must still be combined with a minimum member size constraint. However, it should be noted that the analysis is based on a fixed pixel-like grid, which obviously cannot predict the real stress level very accurately. Hence, it is possible, but in our eyes unlikely, that future work based on adaptive meshing and more advance boundary tracking schemes may change the above conclusion.

Finally, we note that for decreasing stress limit, the volume constraint tends to become inactive, indicating that stress level and not transfer of force from input to output is the governing design factor. Hence, for very low stress levels, the resulting mechanism will be fully distributed compliant and may not be able to transfer a large force from input to output. However, it may still be able to provide large output motions for unloaded output ports.

As stated in the introduction, the purpose of the present work was to study the influence of stress constraints on compliant mechanism design problems. Several conclusions have been drawn based on the study and much insight has been achieved. However, the optimized mechanisms obtained are not relevant for practical applications since the design process was based on linear, small displacement theory. Hence the obvi- ous and most important extension of the work is to include finite deformation theory. Further ideas include adaptive and shape-conforming meshing, extensions to three-dimensional problems, inclusion of buckling constraints and several others. Also, as discussed at several instances, imposing a stress constraint alone is not sufficient to ensure mesh-independency and hence geometrical means, like robust topology optimization formulations (Sigmund, 2009; Wang et al., 2011), must be added as well.

Acknowledgements The authors gratefully acknowledge inspiring discussion and valuable advise from Fengwen Wang and James K. Guest from the Technical University of Denmark and Johns Hopkins University, respectively. Also we would like to acknowledge Niels Aage, from the Technical University of Denmark, for a $\mathrm{C}$ version of MMA as well as valuable technical support.

\section{References}

Allaire G, Jouve F (2008) Minimum stress optimal design with the level set method. Engineering Analysis with Boundary Elements 32:909-918

Allaire G, Jouve F, Maillot H (2004) Topology optimization for minimum stress with the homogeneization method. Structural and Multidisciplinary Optimization 28:87-98

Bourdin B (2001) Filters in topology optimization. International journal for numerical methods in engineering 50:2143-2158

Bruns TE, Tortorelli D (2001) Topology optimization of non-linear elastic structures and compliants mechanisms. Comput Methods Appl Mech Engrg 190:34433459

Cheng G, Jiang Z (1992) Study on topology optimization with stress constraint. Engineering Optimization 20:2:129-148

Cheng GD, Guo X (1997) Epsilon-relaxed approach in structural topology optimization. Structural and Multidisciplinary Optimization 13:258-266

Cook RF (2006) Strength and sharp contact fracture of silicon. J Mater Sci 41:841-872

Díaz A, Sigmund O (1995) Checkerboard patterns in layout optimization. Structural and Multidisciplinary Optimization 10:40-45

Duysinx P, Bendsøe MP (1998) Topology optimization of continuum structures with local stress constraints. International journal for numerical methods in engineering 43:1453-1478

Duysinx P, Sigmund O (1998) New developments in handling stress constraints in optimal material distribution. 7th AIAA/USAF/NASA/ISSMO sympo- 
sium on multidisciplinary analysis and optimization 3:1501-1509

Frecker MI, Ananthasuresh GK, Nishiwaki S, Kota S (1997) Topological synthesis of compliant mechanisms using multi-criteria optimization. Transactions of the ASME 119(2):238-245

Guest JK (2009) Topology optimization with multiple phase projection. Comput Methods Appl Mech Engrg 199:123-135

Guest JK, Prévost JH, Belytschko T (2004) Achieving minimum length scale in topology optimization using nodal design variables and projection functions. International journal for numerical methods in engineering 61:238-254

Guest JK, Asadpoure A, Ha S (2011) Eliminating betacontinuation from heaviside projection and density filter algorithms. Structural and Multidisciplinary Optimization 44:443-453

Howell LL (2013) 21st Century Kinematics, Springer London, chap Compliant mechanisms, pp 189-216. DOI 10.1007/978-1-4471-4510-3

Kirsch U (1990) On singular topologies in optimum structural design. Structural and Multidisciplinary Optimization 2:133-142

Larsen UD, Sigmund O, Bouwstra S (1997) Design and fabrication of compliant micromechanisms and structures with negative poisson's ration. Journal of Microelectromechanical Systems 6:99-106

Le C, Norato J, Bruns T, Ha C, Tortorelli D (2010) Stress-based topology optimization for continua. Structural and Multidisciplinary Optimization 41:605-620

Lee E, Gea HC (2014) A strain based topology optimization method for compliant mechanism design. Structural and Multidisciplinary Optimization 49:199-207

Luo J, Luo Z, Chen S, Tong L, Wang MY (2008) A new level set method for systematic design of hinge-free compliant mechanisms. Comput Methods Appl Mech Engrg 198:318-331

Meneghelli LR, Cardoso EL (2013) Optimization of structures and components, Springer International Publishing Switzerland, chap Design of compliant mechanisms with stress constraints using topology optimization, pp 35-48

Pedersen CBW, Buhl T, Sigmund O (2001) Topology synthesis of large-displacement compliant mechanisms. International Journal of Numerical Methods in Engineering 50(12):2683-2705

Rozvany G, Sobieszczanski-Sobieski J (1992) New optimality criteria methods: forcing uniqueness of the adjoint strains by corner-rounding at constraint intersections. Structural and Multidisciplinary Optimiza- tion 4:244-246

Saxena A, Ananthasuresh G (2001) Topology optimization of compliant mechanisms with strength considerations. Mechanics of Structures and Machines 29(2):199-221, DOI 10.1081/SME-100104480

Sigmund O (1997) On the design of compliant mechanisms using topology optimization. Mech Struct Mach 25(4):493-524

Sigmund O (2001) Design of multiphysics actuators using topology optimization - part i: One-material structures. Comput Methods Appl Mech Engrg 190:6577-6604

Sigmund O (2007) Morphology-based black and white filters for topology optimization. Structural and Multidisciplinary Optimization 33(4-5):401-424

Sigmund O (2009) Manufacturing tolerant topology optimization. Acta Mech Sin 25(2):227-239

Sigmund O, Petersson J (1998) Numerical instabilities in topological optimization: a survey on procedures dealing with checkerboards, mesh-dependencies and local minima. Structural and Multidisciplinary Optimization pp 68-75

Svanberg K (1987) The method of moving asymptotes - a new method for structural optimization. International journal for numerical methods in engineering 24:359-373

Takezawa A, Nishiwaki S, Kitamura M (2010) Shape and topology optimization based on the phase field method and sensitivity analysis. Journal of computational physics 229:2697-2718

Wang F, Lazarov BS, Sigmund O (2011) On projection methods, convergence and robust formulation in topology optimization. Structural and Multidisciplinary Optimization 43:767-784

Zhu B, Zhang X (2012) A new level set method for topology optimization of distributed compliant mechanisms. International journal for numerical methods in engineering 91:843-871

Zhu B, Zhang X, Fatikow S (2014) A multi-objective method of hinge-free compliant mechanism optimization. Structural and Multidisciplinary Optimization 49:431-440 